\title{
Observations of Variable Stars With a Small Telescope at Tabriz University
}

\author{
D. M. Z. Jassur, F. Adabi, \& N. A. Cham \\ Tabriz University, Iran
}

\begin{abstract}
For more than a decade a small group of Astronomers has been monitoring variable stars of different types. In the present contribution I will present the observations of chromospheric active binaries and near contact binaries and the results obtained from their analysis.
\end{abstract}

\section{Introduction}

The short-period group of the RS CVn stars, as defined by Hall (1976) and reviewed by Milano (1981), form a particular subgroup of binary systems that exhibit observational signs of magnetic activity. With orbital periods of less than a day, the stars in short-period group are relatively close to filling their Roche lobes. It has been proposed (e.g., Popper and Ulrich, 1977) that the evolution of RS CVn stars is characterized by at least one of the component stars evolving off the main sequence (but not to the point of Roche overflow). Hence, these stars would appear to be optimal candidates for examining the properties of cool stars prior to some dramatic change in their evolutionary status.

To help in determination of the physical properties of such lower main sequence stars, an analysis of their photometric light curves offers a well-known procedure. However, as has been long recognized (see Wood 1946), the "distortion waves" that are the photometric hallmark of the RS CVn systems complicate a reliable estimation of the system parameters that can be directly read from the optical light curves. These distortion waves do, however, convey information about the active regions of the photosphere. The basic problem in dealing with the photometry of these stars is to extract appropriately all the available information ideally to separate the effects of eclipses and normal proximity interaction from those associated with the "distortions". In this paper we attribute these latter effects to phenomena having methodologically desirable feature of formal representability, which we shall call "Starspots" maculation effects. Our main goals in this paper are to establish an effective procedure and baseline for analyzing the Starspot phenomena on the short-period RS CVn stars to reveal the nature of the activity cycles, and, as well, the physical properties of the stars themselves. 


\section{Observation}

Five systems of the short-period RS CVn type eclipsing binaries, namely XY UMa, UV Psc, CG Cyg, RT And, and ER Vul were photoelectrically observed (e.g., Jassur et al 1993, 1994) during 1989 and 1995. The observations were made in yellow, blue and ultraviolet bands using the 40-cm Cassegrain telescope of Khadjeh Nassir-Addin Observatory of Tabriz University. The light was fed to a single channel photometer tube (RCA 1P21). Figure 1 shows UBV light curves of these stars.

\section{Analysis}

The analysis of light curves was carried out in the following three steps:

(i) The Wilson-Deviney light curve analysis method was used to find uncleaned parameters specifying the system. As much information as possible about each star was gathered from other published data. The values of limb darkening appropriate for the spectral type of respective components of each system were assumed and the available spectroscopic values for mass ratios were retained.

(ii) We deconvolved the theoretical light curve corresponding to our adapted model in the first step from the observed light curve. The object of this step was to obtain the intrinsic light variation of the systems. The quasi-sinusoidal light variation were interpreted in terms of cool star-spots covering a significant fraction of the stellar photosphere.

(iii) We cleaned the original observations by correcting for the presence of the distortion wave, adding the theoretical distortion wave effects of the data with opposite signs. Wilson-Deviney light-curve analysis method was then used to find cleaned parameters to provide us with information about the physical properties of the systems. To show the procedure the analysis of XY UMa is given below as an example.

\section{XY UMa}

$\mathrm{XY} \mathrm{UMa}\left(\mathrm{BD}+55^{\circ} 1317\right)$ is a well known short-period binary system related to the RS CVn group. Geyer has observed it photoelectrically since 1995 and interpreted its unusual photometric behavior as being a result of a large-scale spot activity. The photoelectric observations of this star have been carried out at the Khajeh Nassir-Addin Observatory of Tabriz University and the light curves have been analyzed by the WD method. The results of this analysis are given in Tables Ia, Ib and II.

\section{Near Contact Binaries}

This is a group of binaries (Shaw, 1990) whose components are near enough to each other to have strong proximity effects like W UMA but are not in contact. They are divided into two subclasses according to their physical characteristics: V1010 Ophiuchi and F0 Virginis (named after the brightest member of each class). In former subclasses, the primary is at or near its Roche lobe while the 
secondary is inside its Roche lobe. But in the latter subclasses the primary is inside its Roche lobe and the secondary is at or near Roche lobe. Some members of V1010 Ophiuchis show the $\mathrm{O}^{\prime}$ Connell effect that is max I being brighter than max II. If we accept that the contact binaries evolve from detached systems by means of $\mathrm{AML}$ via magnetic braking or via mass transfer/mass loss, then evidence for this might be the existence of near contact binaries. Our group has carried out observations of some members of near contact binaries (Figure $5 \mathrm{a}$ and 5b.) and some results have been obtained from light curve analysis. The observation and light curve analysis of TZ Lyrae a member of this group are discussed.

\section{TZ Lyrae}

The variable $\mathrm{TZ}$ Lyrae (BD+41 3021 ), an eclipsing binary with a period of 0.5288 days is the brighter component of the visual double ADS 11219. In the NGC of double stars by Aitken (1932) the separation is given as $a=2.87$ " in position angle $358.7^{\circ}$ for the year 1917. D'Esterre (1914) discovered this star but Hoffmeisster determined the correct light elements. A photographic light curve of this Lyrae type system was published by Jordan (1929) from extrafocal images. Using this light curve orbital elements were published by Slonim (1934) and Krat (1935). The only photoelectric observations and light curve of this star were carried out by Binenndijk in 1970 through B and V filters who also obtained geometrical elements of the system from the analysis of his light curves (1972). In 1985, Kalużny has used some normal points extracted from Binenndijk's observations and obtained a solution for this system.

The star was observed through BVR filters using essentially the same equipment employed for the observations of XY UMa. The faint component of the visual double was included in these observations. A total of 224 observations were obtained in each colour. According to our observations there is a significant shift in the epoch of zero phase with respect to the primary minimum $(\mathrm{O}-\mathrm{C}=-$ 0.005 day). The derived light curves of $\mathrm{V}, \mathrm{B}$ and $\mathrm{R}$ colours are illustrated in Figure 5.

Analysis of the light curves was carried out by the differential corrections method. First we assumed that the system is detached and both components are inside their Roche lobes. So we employed mode II with starting values taken from Kalużny (1985). After a few trials we noticed that a detached configuration is not a possible solution. Then we changed to mode IV, thus assuming that the system is a semidetached and the primary component fills its Roche lobe.After some trials the solution started to diverge. Finally mode V was chosen. The final results of this analysis are given in tables IIIa and IIIb. This solution indicates that the secondary component is in contact with its Roche lobe, while the primary is inside its Roche lobe (Figure 7.). The theoretical light curves are shown in Figure 6, overlayed with the observations. 


\section{References}

Aitken, R. G. 1932, New General Catalogue of Double Stars (Carnegie Institution of Washington), 2, 988

Binenndijk, L. 1972, AJ, 77, 7

D'Esterre, C. R. 1914 Astron. Nacher.198, 163

Hall, D. S. 1976, in IAU colloquium 29, Multiple periodic variable stars, ed. W. S. Fitch. P. 287

Jassur, D. M. Z. and Kermani, M. H. 1993 IBVS, No. 3896

Jassur, D. M. Z. and Kermani, M. H. 1994, Astrophys. Space Sci., 219, 35-47

Jordan, F. C. 1929, Allegheny publ. 7, 146

Kaluzny, J. 1985, Acta Astron., Vol.35, 340-341

Krat, W. 1935, Russian Astron. J. 12, 25

Milano, L. 1981, in photometeric and spectroscopic binary systems, ed., E. B. Carling and Z. Kopal.331-360

Popper, D. M. and Ulrich, R.K. 1977, ApJ. (letters), 212, L131

Shaw, J. S. 1990, in Active Close Binary, ed. C. Ibanoglu. P.241

Slonim, E. 1934, Tashkent Bull. No. 4, 85

Wilson R. E., Devinney E. J. 1971, ApJ 166, 605

Wood, F. B. 1946, Contr. Princeton University Obs. No. 21 

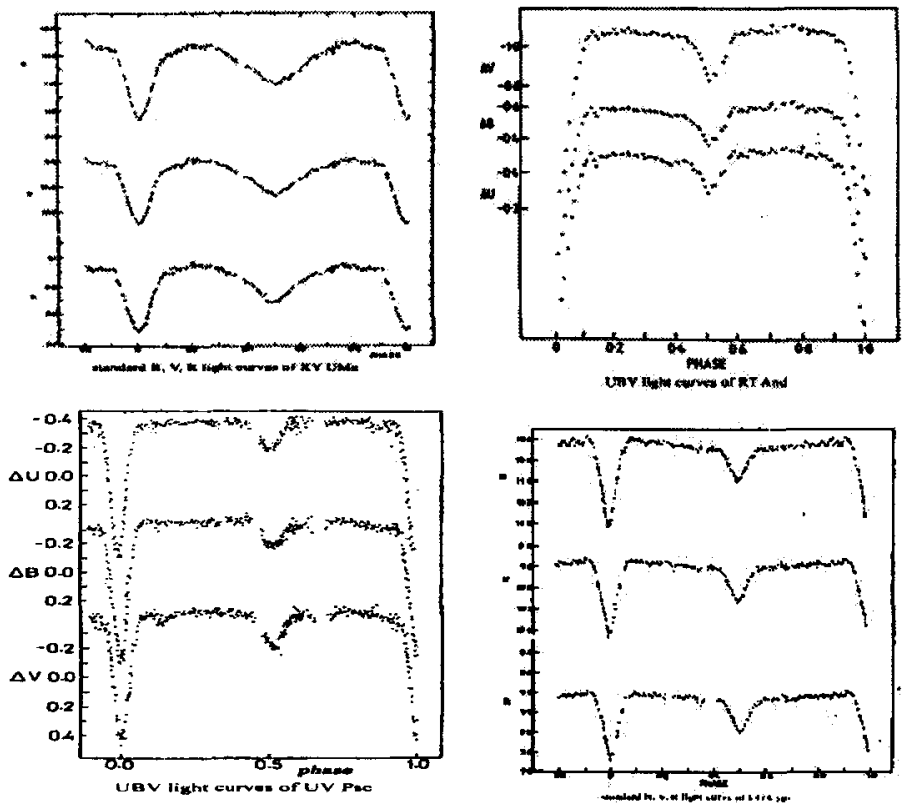

Figure l.light curves of selected short-period RS CVn type eclipsing stars.

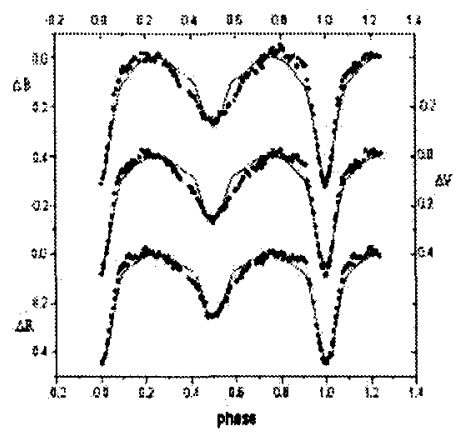

Figure 2. Initial light curve fit of XY UMa. The dots are individual observations. The solid lines are the theoretical light curves based on the parameters in Table $\mathrm{Ia}$ and $\mathrm{Ib}$

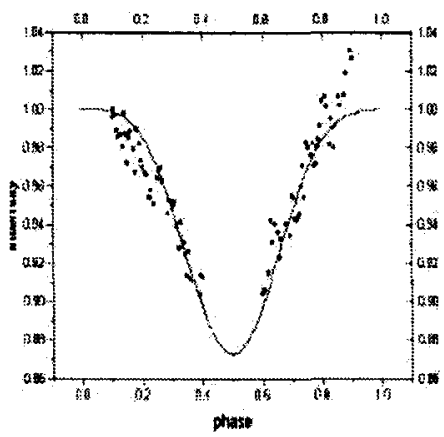

Figure 3. Spot model fit (solid line) to the distortion wave calculation from the difference between the observational points and model in Fig. 2 


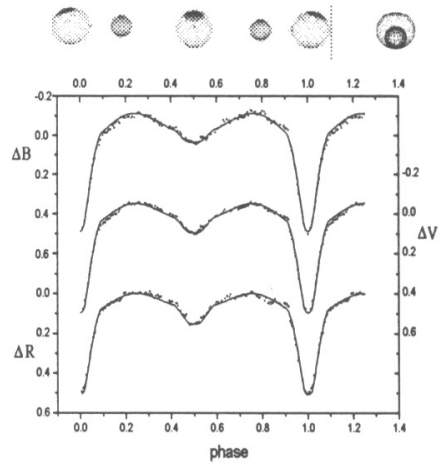

Figure 4. The light curve fit of XY UMa. The dots are individual observation. The solid lines are the theoretical light curves based on the parameters in Table IIa and IIb.

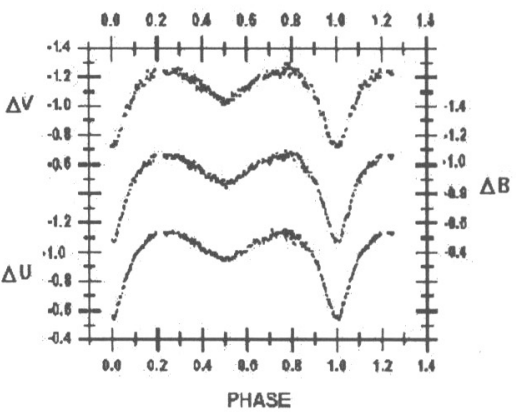

1998 UBV Light curves of BX And

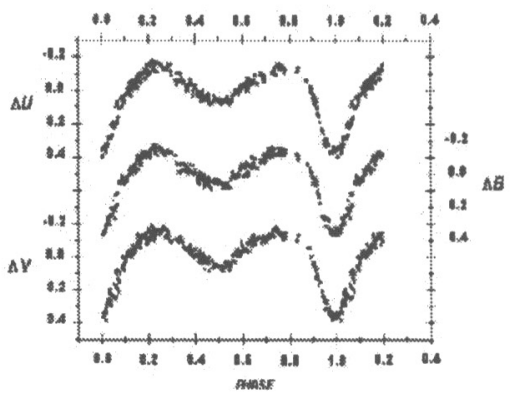

UBV Light curves of ST Aqr

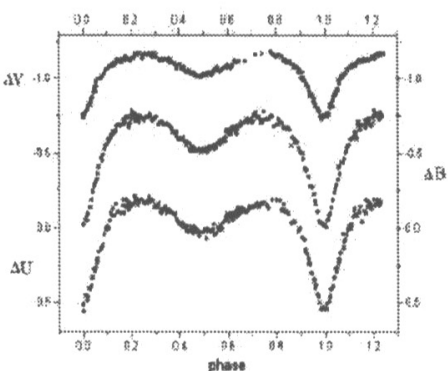

2000 UBV Light curves of BX And

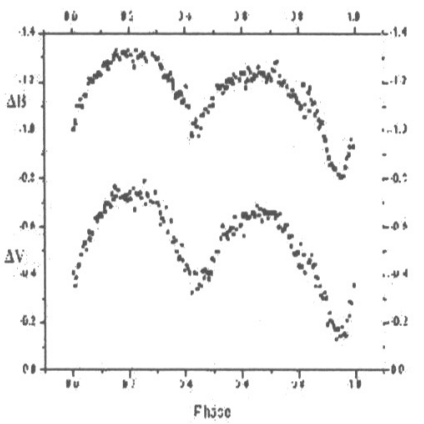

BV Light curves of $\mathrm{CN}$ And

Figure 5a. Light curves of some near contact binaries 


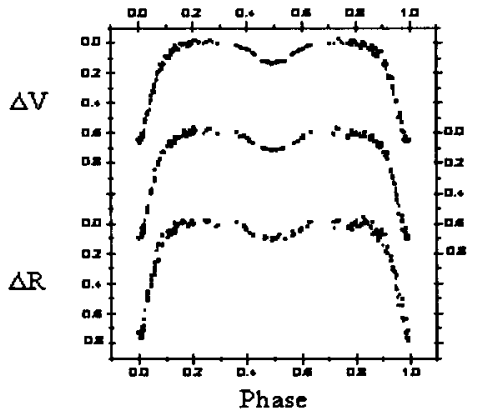

BVR Light curves of TZ Lyrae

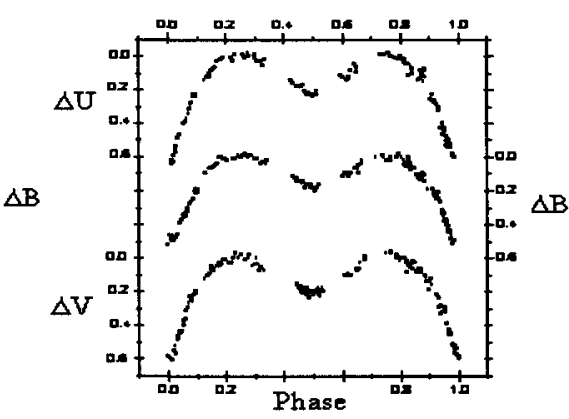

UBV Light curves of GO Cyg

Figure $5 b$. Light curves of some near contact binaries

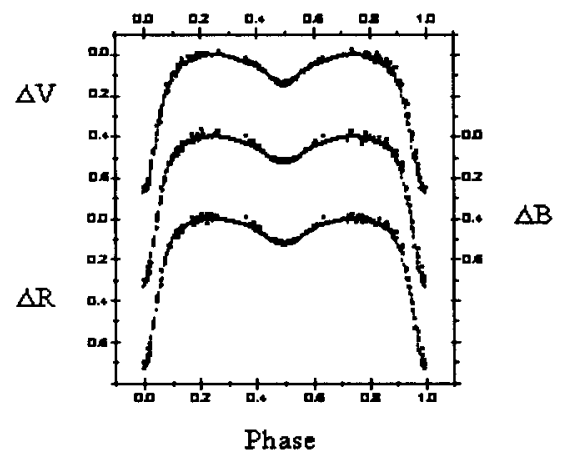

Figure 6.The light curve fit of TZ Lyrea. The dots are individual observations. The solid lines are the theoretical light curves based on the parameters in Table $\mathrm{Va}$ and $\mathrm{Vb}$.

Figure 7.The light curve fit of TZ Lyrea The dots are individual observations. The solid lines are the theoretical light curves based on the parameters in Table Va and Vb.

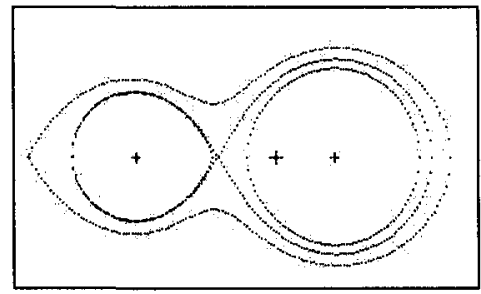


Table Ia (clean light curve)

Photometric Parameters of XY UMa (Wavelength - dependent parameters)

\begin{tabular}{llll}
\hline Parameter & B & V & R \\
\hline$L_{1} /\left(L_{1}+L_{2}\right)$ & $0.9506 \pm 0.0013$ & $0.9219 \pm 0.0011$ & $0.9196 \pm 0.0013$ \\
$L_{2} /\left(L_{1}+L_{2}\right)$ & 0.0494 & 0.0781 & 0.0804 \\
$x_{1}{ }^{*}$ & 0.75 & 0.62 & 0.52 \\
$x_{2}{ }^{*}$ & 0.99 & 0.83 & 0.71 \\
\hline
\end{tabular}

- Not adjusted

Table Ib (clean light curve)

Photometric Parameters of XY UMa (Non-Wavelength-dependent Parameters)

\begin{tabular}{lllll}
\hline Parameter & \multicolumn{1}{c}{$\mathrm{B}$} & $\mathrm{V}$ & $\mathrm{R}$ & \multicolumn{1}{c}{ Average } \\
\hline $\mathrm{i}$ & $82.55^{\circ} \pm 0.20$ & $82.65^{\circ} \pm 0.22$ & $82.45^{\circ} \pm 0.25$ & $82.55^{\circ}$ \\
$\Omega_{1}$ & $3.4250 \pm 0.0068$ & $3.4450 \pm 0.0062$ & $3.4580 \pm 0.0075$ & 3.4427 \\
$\Omega_{2}$ & $4.1960 \pm 0.0155$ & $4.2660 \pm 0.0126$ & $4.3060 \pm 0.0133$ & 4.2560 \\
$\mathrm{~T}_{1}$ & $5872 \pm 35 \mathrm{~K}$ & $5862 \pm 24 \mathrm{~K}$ & $5822 \pm 23 \mathrm{~K}$ & 5852 \\
$\mathrm{~T}_{2}$ & $3965 \pm 13 \mathrm{~K}$ & $3965 \pm 9 \mathrm{~K}$ & $3985 \pm 10 \mathrm{~K}$ & 3972 \\
$q=\frac{m_{2}}{m_{1}}$ & $0.618 \pm 0.002$ & $0.615 \pm 0.002$ & $0.615 \pm 0.003$ & 0.616 \\
$\mathrm{~A}_{1}{ }^{*}$ & 0.5 & 0.5 & 0.5 & 0.5 \\
$\mathrm{~A}_{2}{ }^{*}$ & 0.5 & 0.5 & 0.5 & 0.5 \\
$g_{1}{ }^{*}$ & 0.5 & 0.5 & 0.5 & 0.5 \\
$g_{2}{ }^{*}$ & 0.5 & 0.5 & 0.5 & 0.5 \\
$r_{1}$ (pole) & $0.3518 \pm 0.0008$ & $0.3491 \pm 0.0007$ & $0.3475 \pm 0.0009$ & 0.3495 \\
$r_{1}$ (side) & $0.3654 \pm 0.0010$ & $0.3622 \pm 0.0009$ & $0.3604 \pm 0.0010$ & 0.3627 \\
$r_{1}$ (back) & $0.3810 \pm 0.0011$ & $0.3771 \pm 0.0010$ & $0.3750 \pm 0.0012$ & 0.3777 \\
$r_{1}$ (point) & $0.3969 \pm 0.0015$ & $0.3920 \pm 0.0013$ & $0.3892 \pm 0.0015$ & 0.3927 \\
$r_{2}$ (pole) & $0.2043 \pm 0.0010$ & $0.1989 \pm 0.0008$ & $0.1963 \pm 0.0008$ & 0.1998 \\
$r_{2}$ (side) & $0.2066 \pm 0.0010$ & $0.2010 \pm 0.0008$ & $0.1983 \pm 0.0008$ & 0.2020 \\
$r_{2}$ (back) & $0.2107 \pm 0.0012$ & $0.2047 \pm 0.0009$ & $0.2018 \pm 0.0009$ & 0.2057 \\
$r_{2}$ (point) & $0.2122 \pm 0.0012$ & $0.2060 \pm 0.0009$ & $0.2030 \pm 0.0009$ & 0.2071 \\
\hline
\end{tabular}

*Not adjusted

Table II parameters of the spot

\begin{tabular}{ll}
\hline Colatitude of spot center $\lambda$ & $25^{\circ}$ \\
Longitude of spot center $\beta$ & $180^{\circ}$ \\
Angular radius of the spot $\gamma$ & $9 \%$ \\
Temperature of the spot & $4682^{\circ}$ \\
\hline
\end{tabular}


Table IIIa

Photometric parameters of TZ Lyrae (wavelength-dependent parameters)

\begin{tabular}{llll}
\hline Parameter & B & V & R \\
\hline$L_{4} /\left(L_{1}+L_{2}\right)$ & $0.9557 \pm 0.0132$ & $0.9349 \pm 0.0151$ & $0.9128 \pm 0.0125$ \\
$L_{2} /\left(L_{1}+L_{2}\right)$ & 0.0443 & 0.0651 & 0.0872 \\
$x_{1}{ }^{*}$ & 0.60 & 0.5 & 0.35 \\
$x_{2}{ }^{*}$ & 0.81 & 0.66 & 0.57 \\
\hline \multicolumn{2}{c}{ Not adjusted } & &
\end{tabular}

Table IIIb

Photometric parameters of TZ Lyrae (non-wavelength-dependent parameters)

\begin{tabular}{|c|c|c|c|c|}
\hline Parameter & B & $\mathrm{V}$ & $\mathbf{R}$ & Average \\
\hline$I$ & $78.009 \pm 0.012$ & $77.91 \pm 0.021$ & $77.85 \pm 0.15$ & 77.923 \\
\hline$\Omega_{1}$ & $2.9524 \pm 0.00013$ & $2.9566 \pm 0.0054$ & $2.9517 \pm 0.0046$ & 2.9537 \\
\hline$\Omega_{2}$ & 2.7202 & 2.7251 & 2.7192 & 2.7215 \\
\hline $\mathrm{T}_{1}$ & $8540 \pm 16 k$ & $8592 \pm 17 k$ & $8561 \pm 52 k$ & 8564.3 \\
\hline $\mathrm{T}_{2}$ & $5152 \pm 5 k$ & $5152 \pm 14 k$ & $5153 \pm 18 \mathrm{k}$ & 5152.3 \\
\hline$q=\frac{m_{2}}{m_{1}}$ & $0.4159 \pm 0.0029$ & $0.4168 \pm 0.0041$ & $0.4188 \pm 0.0035$ & 0.4169 \\
\hline $\mathbf{A}_{1}{ }^{*}$ & 1.0 & 1.0 & 1.0 & 1.0 \\
\hline$A_{2} *$ & 0.5 & 0.5 & 0.5 & 0.5 \\
\hline$g_{1} *$ & 0.32 & 0.32 & 0.32 & 0.32 \\
\hline$g_{2} *$ & 0.32 & 0.32 & 0.32 & 0.32 \\
\hline$r_{1}$ (pole) & $0.3951 \pm 0.0014$ & $0.3898 \pm 0.0015$ & $0.3827 \pm 0.0020$ & 0.3892 \\
\hline$r_{1}$ (side) & $0.4061 \pm 0.0017$ & $0.4083 \pm 0.0017$ & $0.4102 \pm 0.0015$ & 0.4082 \\
\hline$r_{1}($ back $)$ & $0.4231 \pm 0.0021$ & $0.4243 \pm 0.0021$ & $0.4254 \pm 0.0055$ & 0.4242 \\
\hline$r_{1}($ point $)$ & $0.4435 \pm 0.0018$ & $0.4445 \pm 0.0028$ & $0.4458 \pm 0.0017$ & 0.4446 \\
\hline$r_{2}$ (pole) & $0.2854 \pm 0.0022$ & $0.2871 \pm 0.0018$ & $0.2818 \pm 0.0037$ & 0.2848 \\
\hline$r_{2}$ (side) & $0.2977 \pm 0.0018$ & $0.2957 \pm 0.0019$ & $0.2967 \pm 0.0011$ & 0.2967 \\
\hline$r_{2}($ back $)$ & $0.3302 \pm 0.0034$ & $0.3161 \pm 0.0030$ & $0.3152 \pm 0.0023$ & 0.3205 \\
\hline$r_{2}$ (point) & $0.3196 \pm 0.0027$ & $0.3167 \pm 0.0039$ & $0.3152 \pm 0.0026$ & 0.3172 \\
\hline
\end{tabular}



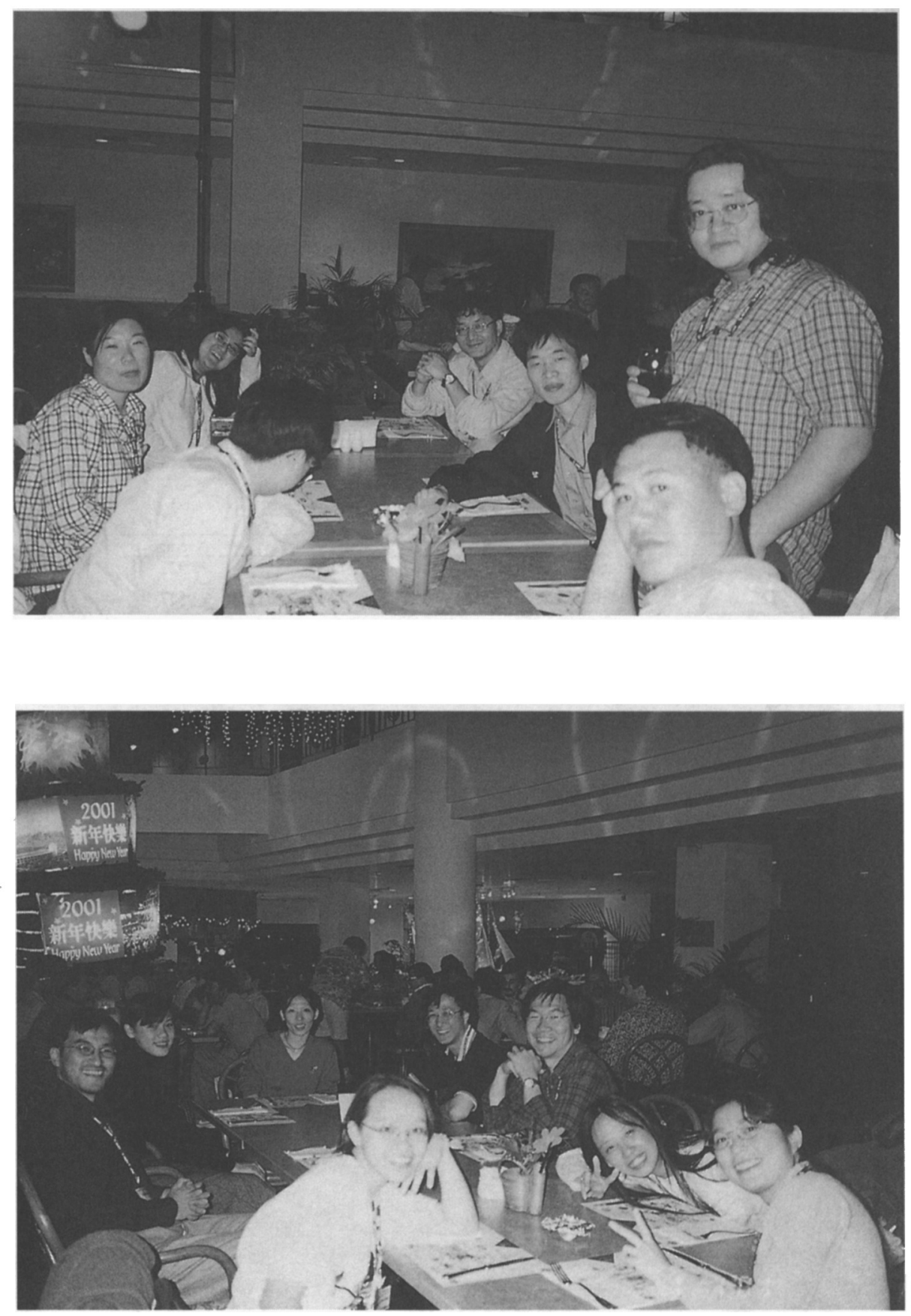

(Top; from left, facing) Woo, Choo, Chun, Kang, Park (standing), Kim (Bottom; from left) H. K. Chang, (Guest of Chang), Peng, Chung, Hwang, Kuan, H. L. Chiou, H. T. Huang 